\title{
Weak-Interacting Holographic QCD
}

\author{
Doron Gazit ${ }^{1, *}$ and Ho-Ung $\mathrm{Yee}^{2,+}$ \\ ${ }^{1}$ Institute for Nuclear Theory, University of Washington, \\ Box 351550, 98195 Seattle, Washington, USA \\ 2 Abdus Salam International Centre for Theoretical Physics, \\ Strada Costiera 11, 34014, Trieste, Italy
}

(Dated: October 24, 2018)

\begin{abstract}
We propose a simple prescription for including low-energy weak-interactions into the framework of holographic QCD, based on the standard AdS/CFT dictionary of double-trace deformations. As our proposal enables us to calculate various electro-weak observables involving strongly coupled QCD, it opens a new perspective on phenomenological applications of holographic QCD. We illustrate efficiency and usefulness of our method by performing a few exemplar calculations; neutron beta decay, charged pion weak decay, and meson-nucleon parity non-conserving (PNC) couplings. The idea is general enough to be implemented in both Sakai-Sugimoto as well as Hard/Soft Wall models.
\end{abstract}

PACS numbers: 


\section{INTRODUCTION}

Holographic QCD is an attempt to construct an effective theory of gauge invariant master fields in large $\mathrm{N}$ limit of QCD. One aspect of it is the existence of an additional space-like dimension roughly corresponding to energy scale of given observables. This is plausible because the large $\mathrm{N}$ limit makes the theory of gauge invariant operators classical, due to large $\mathrm{N}$ factorization, while the concept of renormalization group survives in the limit. Holographic QCD is a theory of gauge invariant fields in 5 dimensions reconciling with these aspects.

Experiences from the known correspondence between $\mathcal{N}=4 \mathrm{SYM}$ in 4 dimensions and Type IIB string theory in $A d S_{5} \times S^{5}$ give us an expectation that the would-be 5-dimensional holographic dual theory of large N QCD becomes highly non-local in UV regime where the corresponding large N QCD is asymptotically free, while we may expect an approximate local theory in IR region as large N QCD becomes strongly coupled. If our interest is only on low-energy observables regarding strongly coupled QCD, it can be a worthwhile approximation to model large N QCD by a local theory in 5 dimensions, as the current models of holographic QCD do. Presumably, it is the UV asymptotic-free region where these local models fail to describe large N QCD properly.

The existing holographic QCD models, like a top-down Sakai-Sugimoto model [1] or a bottom-up Hard/Soft Wall model [2, 3, 4, 5, 6], capture important aspects of low energy QCD such as chiral symmetry and confinement, and explain related experimental observables up to $20 \%$, which one might expect from large N approximation. The previous calculations in the framework concern only pure QCD sector and its electromagnetic couplings (except Ref.[7] whose method is however different from ours). As there are many important processes which involve both weak-interaction and strongly-coupled low energy QCD, it is pertinent to include effective weak-interactions in the framework of holographic QCD, which will allow us a new tool for estimating various hadronic weak processes.

The weak interactions are mediated by exchanges of heavy $W^{ \pm}$or $Z^{0}$ bosons. The tree level weak interactions are thus proportional to the propagators of these bosons. In the low energy limit, in which $q \ll M_{W^{ \pm}, Z^{0}}$, the interactions become effective Fermi point-like vertices. We propose a simple prescription for introducing these effective Fermi vertices of weak-interaction in holographic QCD. In view of the original QCD, this corresponds to 
perturbing the Lagrangian by the effective four-fermi operators

$\Delta \mathcal{L}_{\text {weak }}=\frac{4 G_{F}}{\sqrt{2}}\left(J_{W^{+}} J_{W^{-}}+\cos ^{2} \theta_{W}\left(J_{Z^{0}}\right)^{2}\right)=\frac{4 G_{F}}{\sqrt{2}}\left(\left(J_{L}^{1}\right)^{2}+\left(J_{L}^{2}\right)^{2}+\left(J_{L}^{3}-\sin ^{2} \theta_{W} J_{Q}\right)^{2}\right)(1)$

where $J_{L}^{a}=\sum_{f} \bar{\psi}_{L}^{f} \frac{\sigma^{a}}{2} \psi_{L}^{f}$ and $J_{Q}$ are $S U(2)_{L}$ and electromagnetic currents respectively, and $\theta_{W}$ is the weak angle. When it is necessary, the currents in the above may include leptonic part as well, which should be treated as an external background.

In holographic QCD as a gauge/gravity correspondence, perturbing the QCD Lagrangian would correspond to deforming boundary conditions of 5D fields near the UV boundary in a suitable way. Note that there is a one-to-one map between single-trace[35] operators in QCD and elementary fields of holographic QCD in 5 dimensions. A 5D field $\phi(x, r)$, where $r$ is the 5'th coordinate, behaves near the UV boundary $r \rightarrow \infty$ as $\phi(x, r) \sim c_{1} r^{-\Delta_{-}}+c_{2} r^{-\Delta_{+}}$where $c_{1}$ is non-normalizable and $c_{2}$ is a normalizable mode. For the simplest case of perturbing with a single-trace operator $\mathcal{O}$,

$$
\Delta \mathcal{L}=\int d^{4} x f(x) \mathcal{O}(x)
$$

it is well-known that in the holographic dual theory the boundary condition for the corresponding $5 \mathrm{D}$ field $\phi_{\mathcal{O}}(x, r)$ is modified to be $c_{1}(x)=f(x)$, while the normalizable mode encodes the expectation value of $\mathcal{O}$, that is, $c_{2}(x)=\langle\mathcal{O}(x)\rangle$. However, the perturbation (1) that we are interested in does not belong to this case; it consists of products of two singletrace operators $J^{\mu}$ s. As the elementary fields in holographic 5 dimensions only map to single-trace operators, we can't simply introduce additional 5D fields for these double-trace operators and deform their boundary conditions as above.

There is an answer for this, first proposed for scalar operators [8], and generalized to global symmetry currents [9, 10]. Suppose we perturb by a general polynomial of a single trace operator $\mathcal{O}$,

$$
\Delta \mathcal{L}=\int d^{4} x F(\mathcal{O})
$$

The prescription is that this modifies the boundary condition for the $5 \mathrm{D}$ field $\phi_{\mathcal{O}}$ to be

$$
c_{1}(x)=\left.\frac{\delta F(\mathcal{O})}{\delta \mathcal{O}}\right|_{\mathcal{O} \rightarrow c_{2}(x)},
$$

where the normalization should be such that $c_{2}$ is precisely equal to the expectation value, while $c_{1}$ is the source for $\mathcal{O}$. We will be more specific about precise normalization in our later discussions of concrete examples. 
Although the idea is general enough to be applicable to any model of holographic QCD (see Ref.[11] for a review), we take two prototype examples, the Sakai-Sugimoto model and the Hard/Soft Wall model, to illustrate our proposal. In order to show its usefulness, we analyze a few exemplar physical processes, including the charged pion decay, neutron beta decay, and the leading order parity non-conserving interaction between pions and nucleons. We stress that the prescription we propose should be valid to moderately higher energies before QCD asymptotic freedom sets in.

\section{IMPLEMENTATION}

\section{Sakai-Sugimoto model}

The Sakai-Sugimoto model is based on a string theory set-up of probe $N_{F} D 8$ and $\bar{D} 8$ branes in UV regime being joined at the IR boundary of the background of color $N_{c} D 4$ branes, geometrically realizing spontaneous chiral symmetry breaking $U\left(N_{F}\right)_{L} \times U\left(N_{F}\right)_{R} \rightarrow$ $U\left(N_{F}\right)_{I}$. The resulting configuration can also be viewed as $N_{F} D 8$ branes with two asymptotic UV regions, one for $U\left(N_{F}\right)_{L}$ and the other for $U\left(N_{F}\right)_{R}$. A convenient choice of the 5 'th coordinate is $Z$ ranging from one boundary $Z=-\infty$ to the other $Z=+\infty$, where the IR regime corresponds to near $Z=0$. Holographic dynamics of the chiral symmetry resides in the world volume $U\left(N_{F}\right)$ gauge field $A(x, Z)$ on the $D 8$ branes, whose values near two UV boundaries $Z \rightarrow \pm \infty$ couple to the global $U\left(N_{F}\right)_{L}$ and $U\left(N_{F}\right)_{R}$ currents of QCD respectively. We will focus on the two-flavor $N_{F}=2$ case in the following, but its extension to $N_{F}>2$ is straightforward.

The part of the 5 dimensional action which is relevant for us is

$$
S_{D 8}=-\kappa \int d^{4} x \int d Z \operatorname{tr}\left[\frac{1}{2}\left(1+Z^{2}\right)^{-\frac{1}{3}} F_{\mu \nu}^{2}+\left(1+Z^{2}\right) F_{\mu Z}^{2}\right],
$$

with $\kappa=\frac{\pi}{4} f_{\pi}^{2}=\frac{\lambda N_{c}}{216 \pi^{3}} \simeq 7.45 \times 10^{-3}$ to best fit the experiments for mesons, and the only scale in the model, $M_{K K} \simeq 0.95 \mathrm{GeV}$, is set to 1 . Near the UV boundary $Z \rightarrow \pm \infty$, the second term dominates, from which the equation of motion asymptotes to $\partial_{Z}\left(Z^{2} \partial_{Z} A_{\mu}\right)=0$, and we have

$$
A_{\mu}(Z) \sim A_{\mu}( \pm \infty) \pm \frac{A_{\mu}^{(1)}( \pm \infty)}{Z}+\cdots
$$

near $Z \rightarrow \pm \infty$. As usual, $A_{\mu}(+\infty) \equiv A_{\mu}^{L}$ couples to the $U(2)_{L}$ current $J_{L}^{\mu}$, and similarly for $A_{\mu}(-\infty) \equiv A_{\mu}^{R}$ and $J_{R}^{\mu}$. The sub-leading coefficients $A^{(1)}( \pm \infty)$ are proportional to the 
expectation values of $J_{L, R}$, and to find the precise normalization, we insert (6) in (5) and take a variation with respect to sources $A^{L, R}$ to find the expectation values of $J_{L, R}$. A quick calculation gives us

$$
\eta_{\mu \nu}\left\langle J_{L, R}^{\nu}\right\rangle=-2 \kappa A_{\mu}^{(1)}( \pm \infty)=\mp 2 \kappa \lim _{Z \rightarrow \pm \infty}\left(Z^{2} F_{\mu Z}\right)
$$

where the last expression is a gauge invariant statement.

Having identified the normalized sub-leading expansion of the holographic gauge field $A$, we are ready to implement the effective weak interactions in the set-up. The perturbation (1) contains both quark and lepton currents, and the quark currents are nothing but of the chiral symmetry $U(2)_{L} \times U(2)_{R}$ as the electro-weak interaction is a partial gauging of it. We treat the lepton currents as external backgrounds in the present framework. Recalling that $J_{Q}=J_{L}^{3}+J_{R}^{3}+\frac{1}{6}\left(J_{L}^{U(1)}+J_{R}^{U(1)}\right)$ for quarks with $U(1)$ charge matrix being simply $2 \times 2$ identity, the prescription (44) for our double-trace perturbation (1) gives us the boundary conditions for $A=A^{a} \frac{\sigma^{a}}{2}+A^{U(1)} \mathbf{1}_{2 \times 2}$ near $Z \rightarrow \pm \infty$,

$$
\begin{aligned}
& A_{\mu}^{a}(+\infty)=\frac{8 G_{F}}{\sqrt{2}}\left(-\left.2 \kappa\left(Z^{2} F_{\mu Z}^{a}\right)\right|_{Z \rightarrow+\infty}+J_{L \mu}^{a, \text { lepton }}\right) \\
& A_{\mu}^{a}(-\infty)=0 \quad, \quad a=1,2,
\end{aligned}
$$

from the $W^{ \pm}$vertices, and from the $Z^{0}$ vertex we have

$\frac{1}{1-\sin ^{2} \theta_{W}} A_{\mu}^{3}(+\infty)=\frac{-1}{\sin ^{2} \theta_{W}} A_{\mu}^{3}(-\infty)=\frac{-6}{\sin ^{2} \theta_{W}} A_{\mu}^{U(1)}(+\infty)=\frac{-6}{\sin ^{2} \theta_{W}} A_{\mu}^{U(1)}(-\infty)=K_{\mu}$, with

$$
\begin{aligned}
K_{\mu} & =\frac{8 G_{F}}{\sqrt{2}}\left(-\left.2 \kappa Z^{2}\left(\left(1-\sin ^{2} \theta_{W}\right) F_{\mu Z}^{3}-\frac{\sin ^{2} \theta_{W}}{6} F_{\mu Z}^{U(1)}\right)\right|_{Z \rightarrow+\infty}\right) \\
& +\frac{8 G_{F}}{\sqrt{2}}\left(-\left.2 \kappa \sin ^{2} \theta_{W} Z^{2}\left(F_{\mu Z}^{3}+\frac{1}{6} F_{\mu Z}^{U(1)}\right)\right|_{Z \rightarrow-\infty}+\text { lepton currents }\right)
\end{aligned}
$$

Normally, we are interested in effects which are first order in $G_{F}$, and for that purpose the procedure of applying the above to concrete examples can be simplified in the following way; first solve the normalizable spectrum and wave-functions of the 4D particles of interest without $G_{F}$, which has been done in the previous literature. They will contribute to the right-hand side of (8) and (91) and each mode then entails a non-normalizable piece of first order in $G_{F}$, which enters the calculation of various amplitudes of hadronic weak processes. 
Observe that the non-normalizability issue of the $G_{F}$-perturbed wave-functions is an order $\left(G_{F}\right)^{2}$ question.

\section{Hard/Soft Wall model}

We next implement our prescription in the Hard/Soft Wall model. The Hard/Soft Wall model is a more phenomenological attempt by taking simple AdS space-time with a hard/soft IR cutoff and introducing a minimal set of fields relevant for the chiral symmetry and its breaking. The action takes a form

$$
S_{5 D}=\int d^{4} x \int d z \sqrt{G} e^{-\Phi} \operatorname{tr}\left(-\frac{1}{4 g_{5}^{2}}\left(F_{L, R}\right)^{2}+|D X|^{2}+3|X|^{2}\right),
$$

with $U(2)_{L} \times U(2)_{R}$ gauge fields $A_{L, R}$, a bi-fundamental scalar $X$, a dilaton $\Phi$, and $g_{5}^{2}=$ $\frac{12 \pi^{2}}{N_{c}}=4 \pi^{2}$. One virtue of the model is that a bare quark mass can be introduced via the nonnormalizable profile of $X$, while its normalizable mode parameterizes chiral condensate. A popular coordinate system often used is $d s^{2}=\frac{1}{z^{2}}\left(-d z^{2}+d x_{\mu} d x^{\mu}\right)$ where the UV boundary is $z \rightarrow 0$. As the dilaton in the Soft Wall model is $\Phi(z)=z^{2} \rightarrow 0$ near $z \rightarrow 0$, the UV dynamics of the Hard and Soft Wall models is identical, and so is our implementation of weak interactions via perturbed UV boundary conditions.

Due to the expectation value $\langle X\rangle=\frac{1}{2}\left(m_{q} z+\sigma z^{3}\right) \mathbf{1}_{2 \times 2}=v(z) \mathbf{1}_{2 \times 2}$ which breaks the axial part of the chiral symmetry $A \equiv \frac{1}{2}\left(A_{L}-A_{R}\right)$, there appear mixed kinetic terms between $A_{M}=\left(A_{\mu}, A_{z}\right)$ and the phase part $P$ of $X=\langle X\rangle e^{i P}$, which is typical in a Higgs mechanism,

$$
S_{\text {mixing }}=-2 \int d^{4} x d z \frac{1}{z} \operatorname{tr}\left(\frac{1}{g_{5}^{2}}\left(\partial_{z} A_{\mu}\right)\left(\partial^{\mu} A_{z}\right)+\frac{2 v^{2}}{z^{2}} A_{\mu}\left(\partial^{\mu} P\right)\right),
$$

where the indices are contracted with flat metric $\eta_{\mu \nu}=\operatorname{diag}(+1,-1,-1,-1)$. This brings a slight complication into the analysis of UV behavior of our 5D gauge fields, but not in an essential way as we will see shortly. Note that the isospin part of the chiral symmetry $V=\frac{1}{2}\left(A_{L}+A_{R}\right)$ remains intact, and the asymptotic UV expansion from equation of motion is easily found to be

$$
V_{\mu} \rightarrow V_{\mu}(0)+V_{\mu}^{(1)} z^{2}+\cdots,
$$

and the usual interpretation of source and expectation value goes as before.

To study the $\left(A_{\mu}, A_{z}, P\right)$ system clearly, it is convenient to work in a $5 \mathrm{D}$ analog of $R_{\xi^{-}}$ gauge by adding the following gauge fixing term in the bulk [12, 13],

$$
S_{g . f .}=-\frac{1}{2 \xi} \int d^{4} x d z \frac{1}{z} \operatorname{tr}\left[\partial_{\mu} A^{\mu}-2 \xi\left(\frac{1}{g_{5}^{2}} z \partial_{z}\left(\frac{A_{z}}{z}\right)-\frac{2 v^{2}}{z^{2}} P\right)\right]^{2}
$$


which removes the mixing terms between $A_{\mu}$ and $\left(A_{z}, P\right)$, up to an important left-over boundary term,

$$
S_{b d r y}=\frac{2}{g_{5}^{2}} \int d^{4} x \operatorname{tr}\left(\left.\frac{1}{z} A_{\mu} \partial^{\mu} A_{z}\right|_{z \rightarrow 0}\right) .
$$

This boundary term will be important later in discussing coupling of external sources to the pions. The above gauge is especially useful in identifying physical degrees of freedom in a transparent way. Upon $4 \mathrm{D}$ reduction via $\mathrm{KK}$ mode expansion, one combination of $A_{z}$ and $P$ gives longitudinal components of massive vector mesons from $A_{\mu}$, while the other contains the pions and the excited scalar mesons. To separate longitudinal components of vector mesons from the pions, we further take a unitary gauge $\xi \rightarrow \infty$, upon which $\left(A_{z}, P\right)$ includes only physical pions and its excitations, and allows us to replace $P$ with $A_{z}$ via

$$
\frac{1}{g_{5}^{2}} z \partial_{z}\left(\frac{A_{z}}{z}\right)=\frac{2 v^{2}}{z^{2}} P
$$

The resulting equation of motion for $A_{z}$ can be solved for the pions and excited scalar mesons, with the UV boundary condition $A_{z} \sim \mathcal{O}(z)$ for normalizability [13].

The action for $A_{\mu}$ in our gauge then becomes simply

$$
S_{A}=\int d^{4} x d z \frac{1}{z} \operatorname{tr}\left(-\frac{1}{2 g_{5}^{2}}\left(F_{\mu \nu}\right)^{2}+\frac{1}{g_{5}^{2}}\left(\partial_{z} A_{\mu}\right)^{2}+\frac{4 v^{2}(z)}{z^{2}} A_{\mu} A^{\mu}\right)+S_{b d r y},
$$

where indices are contracted with the flat metric $d s^{2}=-d z^{2}+d x^{2}$, and its equation of motion along $z$ is

$$
\partial_{z}\left(\frac{1}{z} \partial_{z} A_{\mu}\right)-\frac{1}{z} \partial_{\nu} F_{\nu \mu}-\frac{4 g_{5}^{2} v^{2}(z)}{z^{3}} A_{\mu}=0
$$

whose solution has a UV asymptotic

$A_{\mu} \sim c_{1} z K_{1}\left(g_{5} m_{q} z\right)+c_{2} z I_{1}\left(g_{5} m_{q} z\right) \sim A_{\mu}^{(0)}+A_{\mu}^{(1)} z^{2}+\frac{1}{2}\left(\partial_{\nu} F_{\nu \mu}^{(0)}+g_{5}^{2} m_{q}^{2} A_{\mu}^{(0)}\right) z^{2} \log z+\cdots$

which is essentially same to the case without a Higgs breaking. We observe that the UV behavior is not much affected by the symmetry breaking through $\langle X\rangle \neq 0$, and we can apply the usual dictionary of source and expectation value to $A_{\mu}^{(0)}$ and $A_{\mu}^{(1)}$ respectively. It is also possible to prove this assertion more rigorously by the standard procedure of holographic renormalization [14]. Observe that the previously identified boundary term (14) in our unitary gauge also contributes to the expectation value as it becomes

$$
S_{b d r y}=\frac{2}{g_{5}^{2}} \int d^{4} x \operatorname{tr}\left(\left.A_{\mu}^{(0)}\left(\frac{1}{z} \partial^{\mu} A_{z}\right)\right|_{z \rightarrow 0}\right)
$$


and there is a similar term for $V=\frac{1}{2}\left(A_{L}+A_{R}\right)$ too.

As we find that the usual gauge/gravity dictionary holds for the $U(2)_{L} \times U(2)_{R}$ bulk gauge fields, it is straightforward to implement our proposal for effective weak interactions. The precise relation between $A_{L, R}^{(1)}$ and $\left\langle J_{L, R}\right\rangle$ from the above discussion is

$$
\eta_{\mu \nu}\left\langle J_{L, R}^{\nu}\right\rangle=-\frac{2}{g_{5}^{2}} A_{\mu}^{(1) L, R}+\left.\frac{1}{g_{5}^{2}} \frac{1}{z} \partial_{\mu} A_{z}^{L, R}\right|_{z \rightarrow 0}=\left.\frac{1}{g_{5}^{2}} \frac{1}{z} F_{\mu z}^{L, R}\right|_{z \rightarrow 0}
$$

where the second contribution from (18) makes the final result gauge invariant, which is important to include the cases with the pions coming from $A_{z} \sim \mathcal{O}(z)$. Using this, the Fermi-interaction via $W^{ \pm}$exchange corresponds to the boundary condition

$$
\begin{aligned}
& A_{L \mu}^{a}(0)=\frac{8 G_{F}}{\sqrt{2}}\left(\left.\frac{1}{g_{5}^{2}} \frac{1}{z} F_{\mu z}^{L a}\right|_{z \rightarrow 0}+J_{L \mu}^{a, \text { leptons }}\right), \quad a=1,2, \\
& A_{R \mu}^{a}(0)=0,
\end{aligned}
$$

and similarly for the $Z^{0}$ exchange as in (9).

The boundary conditions (8), (91) and (20) are our main points in the paper. To find effects which are lowest order in $G_{F}$, we first solve normalizable modes dynamics without $G_{F}$, and via (20) non-normalizable perturbations of linear order in $G_{F}$ are induced. We point out that to leading order in $G_{F}$, our prescription is equivalent to large $\mathrm{N}$ factorizing our effective $J J$ weak vertices and then calculating chiral form factors of hadrons.

\section{EXEMPLAR CALCULATIONS}

(1) Charged pion weak decay $\pi^{+} \rightarrow \mu^{+} \nu_{\mu}$

For an illustrative purpose of showing how the prescription works, we compute via our method a few simple hadronic weak processes which involve only $W^{ \pm}$exchange, while the analysis of neutral current phenomena and fitting the weak angle $\theta_{W}$ are left to the future.

One of the cleanest observables from charged current exchange is the decay of charged pions; more than $99 \%$ of $\pi^{+}$decay to $\mu^{+} \nu_{\mu}$ via $W^{+}$-exchange, while the electron channel $\pi^{+} \rightarrow e^{+} \nu_{e}$ is helicity suppressed by $\frac{m_{e}^{2}}{m_{\mu}^{2}} \sim 10^{-5}$. Computing the relevant pion coupling

to the external leptonic current $J_{L}^{+, \text {lepton }}=\left(J_{L}^{1}-i J_{L}^{2}\right)^{\text {lepton }}=\bar{l}_{L} \gamma^{\mu} \nu_{L}$ is in fact easy as the lepton current simply acts as a source for the corresponding quark current through (8) in the Sakai-Sugimoto model, or (20) for the Hard/Soft Wall model. Expanding $A^{a} \frac{\sigma^{a}}{2}=$ 
$\frac{1}{\sqrt{2}}\left(A^{+} \sigma^{-}+A^{-} \sigma^{+}\right)$for bulk fields [36], this is equivalent to having an external source

$$
\begin{aligned}
A^{+}(+\infty) & \left.=\left(4 G_{F}\right) J_{L}^{+, \text {lepton }}=\left(4 G_{F}\right) \bar{l}_{L} \gamma^{\mu} \nu_{L} \quad \text { (Sakai }- \text { Sugimoto }\right) \\
A_{L}^{+}(0) & =\left(4 G_{F}\right) \bar{l}_{L} \gamma^{\mu} \nu_{L} \quad(\text { Hard } / \text { Soft Wall })
\end{aligned}
$$

We consider the case of Sakai-Sugimoto model first. To identify how pions interact with the above external source for chiral currents, working in the $R_{\xi}$-gauge with unitary limit $\xi \rightarrow \infty$ again seems most convenient. By the similar procedure to reach (16) in the Hard/Soft Wall models, the final action in our unitary gauge becomes

$$
\begin{aligned}
S= & -\kappa \int d^{4} x \int d Z \operatorname{tr}\left[\frac{1}{2}\left(1+Z^{2}\right)^{-\frac{1}{3}} F_{\mu \nu}^{2}-\left(1+Z^{2}\right)\left(\partial_{Z} A_{\mu}\right)^{2}\right] \\
& +\int d^{4} x \operatorname{tr}\left(\partial_{\mu} \pi\right)^{2}-f_{\pi} \operatorname{tr}\left[\left.\left(\partial^{\mu} \pi\right) A_{\mu}\right|_{-\infty} ^{+\infty}\right],
\end{aligned}
$$

where we used $A_{Z}=\frac{1}{\sqrt{\pi \kappa}} \frac{1}{1+Z^{2}} \pi(x)$ in our unitary gauge with $\kappa=\frac{\pi}{4} f_{\pi}^{2}$. Note that the last term comes from the remaining boundary term after adding the gauge fixing term as in (14). Mode expansion of $A_{\mu}$ contains (pseudo) vector mesons as normalizable modes plus external sources for the chiral currents as non-normalizable modes. These non-normalizable modes then couple to the pions only through the last boundary term that we are looking for. The result in fact agrees with the old current algebra expression. Expanding $\pi=$ $\frac{1}{\sqrt{2}}\left(\pi^{+} \sigma^{-}+\pi^{-} \sigma^{+}\right)$and plugging in $A(+\infty)=\frac{1}{\sqrt{2}} A^{+}(+\infty) \sigma^{-}=\frac{4 G_{F}}{\sqrt{2}}\left(\bar{l}_{L} \gamma^{\mu} \nu_{L}\right) \sigma^{-}$, we get the coupling

$$
\mathcal{L}_{\pi \bar{l} \nu}=-2 G_{F} f_{\pi}\left(\partial_{\mu} \pi^{-}\right)\left(\bar{l}_{L} \gamma^{\mu} \nu_{L}\right)
$$

We expect the same result from the Hard/Soft Wall model as well. Since in our gauge the pions reside in $A_{z}=\pi(x) f(z)$ with a normalized profile $f(z) \sim z$, the previously identified boundary term (14) in the unitary gauge gives us the same type of coupling between $\pi(x)$ and $A_{L}^{+}(0)=\left(4 G_{F}\right) \bar{l}_{L} \gamma^{\mu} \nu_{L}$. To realize this, note that $A=\frac{1}{2}\left(A_{L}-A_{R}\right)$, thus the boundary term (14) reproduces

$$
\left(\left.\frac{1}{g_{5}^{2} z} f(z)\right|_{z \rightarrow 0}\right) \operatorname{tr}\left(\left.\left(\partial_{\mu} \pi\right)\left(A_{L}^{\mu}-A_{R}^{\mu}\right)\right|_{z \rightarrow 0}\right) \equiv-f_{\pi} \operatorname{tr}\left(\left.\left(\partial_{\mu} \pi\right)\left(A_{L}^{\mu}-A_{R}^{\mu}\right)\right|_{z \rightarrow 0}\right),
$$

so that we have a near $z \rightarrow 0$ expansion of the pion profile $A_{z}=\pi(x) f(z)$,

$$
f(z)=-\left(g_{5}^{2} f_{\pi}\right) z+\cdots,
$$


which will be useful for later calculations involving the pions. Recall that the coupling strength of the pions to external current sources, as appears in Eq. (24) is one of the definitions of $f_{\pi}$. Using standard kinematics [30], one uses the experimental decay rate to find $f_{\pi} \approx 92 \mathrm{MeV}$. Thus, in weak-interacting holographic QCD we successfully predict not only the operator structure but also the value of the decay constant.

\section{(2) Neutron beta decay}

We next analyze a more complicated weak-process involving nucleons; neutron beta decay to proton and the first generation of leptons, $n \rightarrow p^{+} e^{-} \overline{\nu_{e}}$, with $\sim 100 \%$ branching ratio. As we treat the lepton current $J_{L}^{- \text {, lepton }}=\overline{\nu_{L}} \gamma^{\mu} e_{L}$ as external, the analysis is similar to the previous example, that is, the lepton current will play a role of external source for the chiral symmetry current

$$
\begin{aligned}
A^{-}(+\infty) & \left.=\left(4 G_{F}\right) \overline{\nu_{L}} \gamma^{\mu} e_{L} \quad \text { (Sakai - Sugimoto }\right) \\
A_{L}^{-}(0) & =\left(4 G_{F}\right) \overline{\nu_{L}} \gamma^{\mu} e_{L} \quad(\text { Hard } / \text { Soft Wall })
\end{aligned}
$$

and we need how the nucleons couple to the above external source of chiral currents. As a model calculation, we proceed by adopting the recent effective field approach to holographic baryons in Ref.[15, 16] for the Sakai-Sugimoto model, and in Ref.[12] for the Hard Wall model. See also Refs.[17, 18, 19, 20, 21, 22, 23] for other approaches to holographic baryons.

One difference from the previous example of the pions is that the resulting coupling would be momentum-dependent on general grounds. In other words, the result should be phrased as a form factor [24, 25, 26, 27]. The resulting form factor in general can be rewritten as a sum over infinite number of intermediate excitations, similar in spirit to vector dominance [26]. The case of pions in the previous example is special in this respect as they couple to the bulk gauge fields only at the boundary through $S_{b d r y}$ [37]. As the neutron beta decay is a 3-body decay, the relevant momentum transfer is the invariant mass square $q^{2}$ of the $e^{-} \overline{\nu_{e}}$ pair, which ranges $m_{e}^{2}<q^{2}<\left(m_{n}-m_{p}\right)^{2}$. In practice however the relevant $q^{2}$ in our case is too small compared to the QCD scale to make any notable difference from simply using $q^{2}=0$ in numerical computations.

We work in the Sakai-Sugimoto model first. In our previous unitary gauge, we solve the equation of motion for $A_{\mu}$ of a specific 4-momentum $q^{\mu}$ with our prescribed boundary condition $A_{\mu}(+\infty)=\frac{8 G_{F}}{\sqrt{2}} J_{L \mu}^{\text {lepton }}$ and $A_{\mu}(-\infty)=0$. Writing $A_{\mu}(x, Z)=A_{\mu}(Z) e^{-i q \cdot x}$, the equation for $A_{\mu}(Z)$ in fact splits into two parts, one for transverse and the other for longitudinal 
polarization, and the final form of the solution is

$$
A_{\mu}=A^{T}(q, Z)\left[\eta_{\mu \nu}-\frac{q_{\mu} q_{\nu}}{q^{2}}\right] A^{\nu}(+\infty)+\frac{q_{\mu}}{q^{2}}(q \cdot A(+\infty)) \psi_{+}(Z)
$$

with $A^{T}(q, Z)$ satisfying

$$
q^{2}\left(1+Z^{2}\right)^{-\frac{1}{3}} A^{T}+\partial_{Z}\left(\left(1+Z^{2}\right) \partial_{Z} A^{T}\right)=0
$$

with the boundary condition $A^{T}(q,+\infty)=1, A^{T}(q,-\infty)=0$, and $\psi_{+}(Z)=\frac{1}{2}(1+$ $\left.\frac{2}{\pi} \tan ^{-1}(Z)\right)$. By computing how holographic baryons couple to the above $q^{2}$-dependent bulk gauge field, we read off nucleon interaction with an external source $A_{\mu}(+\infty)$, which in our case is an external lepton current.

This is not the whole story however. There is an additional important contribution coming from tree-level pion-exchange. This is because pions couple to external currents by the last term in (23), and from the interaction of holographic baryons to $A_{Z}$ in the bulk, we can also obtain an axial coupling of pions to the nucleons, and hence follows a coupling between external sources and the nucleons via tree-level pion-exchange.

For a practical computation in the following, we should be specific about a model of holographic baryons, for which we choose the model in Ref.[15] as an example. The effective action of a spin- $\frac{1}{2}, S U(2)$ doublet holographic baryon $\mathcal{B}$, as the lowest spin state of collective quantization of $5 \mathrm{D}$ instanton-baryon on the $N_{F}=2 D 8$ branes, is most easily written in the conformal coordinate system $\left(x^{\mu}, w\right)$ with $w=\int_{0}^{Z} \frac{d Z^{\prime}}{\left(1+Z^{\prime 2}\right)^{\frac{2}{3}}}$, under which the 5D baryon action doesn't involve spin connections,

$$
S_{\text {baryon }}=\int d^{4} x \int d w\left[i \overline{\mathcal{B}} \gamma^{M}\left(\partial_{M}-i A_{M}\right) \mathcal{B}-m_{B}(w) \overline{\mathcal{B}} \mathcal{B}+(0.90) i \overline{\mathcal{B}} \gamma^{M N} F_{M N} \mathcal{B}+\cdots\right]
$$

where the position dependent mass term $m_{B}(w)$ can be obtained from the energy of the $S^{4}$-wrapped $D 4$ brane at the position $w$, whose explicit form is not essential in our presentation, and the last term is determined to reproduce the long-range tail of the instanton-baryon solution [38]. We then perform usual KK reduction to 4D by expanding $\mathcal{B}(x, w)=f_{L}(w) N_{L}(x)+f_{R}(w) N_{R}(x)$, where $\left(N_{L}, N_{R}\right) \equiv N(x)$ is the 4D baryon mode we identify as the nucleons, and the profile functions $f_{L, R}(w)$ satisfy a suitable eigen-value equation with the eigenvalue $m_{N}$ being the mass of the nucleons. We have an important parity property $f_{R}(-w)=f_{L}(w)$. Once we find $f_{L, R}$, we can insert our previous $A_{\mu}$ from (29) and $A_{Z}$ into the action (31) to find how external sources and the pions couple to the nucleons. 
After a straightforward calculation, the result is summarized by [15, 29]

$$
\mathcal{L}_{N-\text { external }}=\bar{N} \gamma^{\mu} \mathcal{V}_{\mu} N+\bar{N} \gamma^{\mu} \gamma^{5} \mathcal{A}_{\mu} N+\bar{N} \gamma^{\mu \nu} \mathcal{F}_{\mu \nu} N
$$

with

$$
\begin{aligned}
\mathcal{V}_{\mu} & =\frac{1}{2} \int d w\left(\left|f_{L}\right|^{2}+\left|f_{R}\right|^{2}\right) A_{\mu}(w)+(0.90) \int d w\left(\left|f_{L}\right|^{2}-\left|f_{R}\right|^{2}\right) \partial_{w} A_{\mu}(w), \\
\mathcal{A}_{\mu} & =\frac{1}{2} \int d w\left(\left|f_{L}\right|^{2}-\left|f_{R}\right|^{2}\right) A_{\mu}(w)+(0.90) \int d w\left(\left|f_{L}\right|^{2}+\left|f_{R}\right|^{2}\right) \partial_{w} A_{\mu}(w), \\
\mathcal{F}_{\mu \nu} & =(0.54) i \int d w f_{L} f_{R} F_{\mu \nu}(w),
\end{aligned}
$$

for the nucleon couplings to external sources encoded in (29), and

$$
\mathcal{L}_{N-\pi}=-\frac{g_{A}}{f_{\pi}} \bar{N} \gamma^{\mu} \gamma^{5}\left(\partial_{\mu} \pi\right) N
$$

with

$$
g_{A}=2 \int d w\left|f_{L}\right|^{2} \psi_{0}(w)+4 \cdot(0.90) \int d w\left|f_{L}\right|^{2}\left(\frac{d \psi_{0}}{d w}\right),
$$

for the pion-nucleon axial coupling. Numerical evaluation gives $g_{A} \simeq 1.3$, which compares well with experiment $g_{A}^{e x p}=1.27[15]$.

Although we can solve (30) for $A^{T}$ numerically for every $q^{2}$ of a problem to find $A_{\mu}(w)$ inserted in the above result, it is a good approximation in our case to take the leading small $q^{2}$ expansion as $q^{2} \ll 1$, which gives a simple formula

$$
\mathcal{V}_{\mu}=\frac{1}{2} A_{\mu}(+\infty) \quad, \quad \mathcal{A}_{\mu}=\frac{g_{A}}{2} A_{\mu}(+\infty)
$$

with the exactly same $g_{A}$ as in (35), and numerically we have

$$
\mathcal{F}_{\mu \nu}=\frac{1}{2} \cdot(0.90) i\left(\int d w f_{L} f_{R}(w)\right) F_{\mu \nu}(+\infty) \simeq(0.42) i F_{\mu \nu}(+\infty) \quad .
$$

Interestingly, it can be easily checked that the contribution from the tree-level pion exchange has the similar shape to the one from the $\mathcal{A}_{\mu}$ above,

$$
\mathcal{L}_{\pi-\text { exchange }}=-\frac{g_{A}}{2} \frac{q_{\mu} q_{\nu}}{q^{2}} \bar{N} \gamma^{\mu} \gamma^{5} A^{\nu}(+\infty) N
$$

so that the sum of the all contributions is

$$
\begin{aligned}
\mathcal{L}_{N-\text { external }} & =\frac{1}{2} \bar{N} \gamma^{\mu} A_{\mu}(+\infty) N+\frac{g_{A}}{2}\left(\eta_{\mu \nu}-\frac{q_{\mu} q_{\nu}}{q^{2}}\right) \bar{N} \gamma^{\mu} \gamma^{5} A^{\nu}(+\infty) N \\
& +(0.42) i \bar{N} \gamma^{\mu \nu} F_{\mu \nu}(+\infty) N+\mathcal{O}\left(q^{2}\right)
\end{aligned}
$$


Expanding $A_{\mu}(+\infty)=\frac{1}{\sqrt{2}} A^{-}(+\infty) \sigma^{+}=\frac{4 G_{F}}{\sqrt{2}}\left(\overline{\nu_{L}} \gamma^{\mu} e_{L}\right) \sigma^{+}$and $N=(p, n)^{T}$, we finally have

$$
\mathcal{L}_{\bar{n} p e^{-} \bar{\nu}_{e}}=\frac{4 G_{F}}{\sqrt{2}}\left[\frac{1}{2}\left(\bar{n} \gamma_{\mu} p\right)+\frac{g_{A}}{2}\left(\eta_{\mu \nu}-\frac{q_{\mu} q_{\nu}}{q^{2}}\right)\left(\bar{n} \gamma^{\nu} \gamma^{5} p\right)-(0.84) q^{\nu}\left(\bar{n} \gamma_{\mu \nu} p\right)\right]\left(\overline{\nu_{L}} \gamma^{\mu} e_{L}\right),(40
$$

for the effective vertex of the neutron beta decay. Note that the Sakai-Sugimoto model does not have a bare quark mass by construction and the pions are massless [39]. In any realistic application of the above result, we therefore should replace the $q^{2}$-pole in the above with $m_{\pi}^{2}$. This unsatisfactory feature will be absent in the Hard Wall model however, as we can introduce a bare quark mass in the set-up.

We next turn to the analysis in the Hard Wall model. As the procedure is identical to the previous one in the Sakai-Sugimoto model, we will be brief in explaining the intermediate steps while showing the results. For our purpose we will work in the holographic baryon model of Ref.[12] for the Hard Wall model as it is most suitable to see how holographic baryons couple to external chiral currents. The holographic baryon action for the Hard Wall model reads as

$$
\begin{aligned}
S_{N} & =\int d^{4} x \int d z \sqrt{G_{5}}\left[i \bar{N}_{1} \Gamma^{M} D_{M} N_{1}+i \bar{N}_{2} \Gamma^{M} D_{M} N_{2}-m_{5} \bar{N}_{1} N_{1}+m_{5} \bar{N}_{2} N_{2}\right] \\
& +\int d^{4} x \int d z \sqrt{G_{5}}\left[-g \bar{N}_{1} X N_{2}-g \bar{N}_{2} X^{\dagger} N_{1}\right] \\
& +\int d^{4} x \int d z \sqrt{G_{5}}\left[(i D)\left(\bar{N}_{1} \Gamma^{M N} F_{M N}^{L} N_{1}-\bar{N}_{2} \Gamma^{M N} F_{M N}^{R} N_{2}\right)\right]
\end{aligned}
$$

where $N_{1}\left(N_{2}\right)$ is a doublet under $S U(2)_{L}\left(S U(2)_{R}\right)$. The $5 \mathrm{D}$ Dirac mass $m_{5}$ is related to the scaling dimension $\Delta$ of the QCD 3-quark nucleon operator by $m_{5}=(\Delta-2)$, and for simplicity we take $\Delta=\frac{9}{2}$. The second line gives us the observed nucleon mass $m_{N}=$ $0.94 \mathrm{GeV}$ when the chiral symmetry is broken $\langle X\rangle \neq 0$. For the popular values $z_{m}=$ $(330 \mathrm{MeV})^{-1}, \sigma=(311 \mathrm{MeV})^{3}$ and $m_{q}=2.34 \mathrm{MeV}$, this determines the coupling $g$ to be $g=9.18$. We leave the coefficient $D$ as a free parameter. For these parameters, we have $f_{\pi}=0.084 \mathrm{GeV}$ and the pion-nucleon axial coupling is numerically $g_{A}=(0.33+1.02 D)$ where $D$ is in units of $(\mathrm{GeV})^{-1}$.

As the tree-level pion-exchange contribution is given by (38) except the $q^{2}$-pole replaced by $m_{\pi}^{2}$, it is an order $\mathcal{O}\left(q^{2}\right)$ effect which is negligible. By essentially same procedures as in the Sakai-Sugimoto case, our final effective interaction for the neutron beta decay in the Hard Wall model is found to be

$$
\mathcal{L}_{\bar{n} p e^{-} \bar{\nu}_{e}}=\frac{4 G_{F}}{\sqrt{2}}\left[\frac{1}{2}\left(\bar{n} \gamma_{\mu} p\right)+\frac{1}{2}(0.33+1.02 D)\left(\bar{n} \gamma_{\mu} \gamma^{5} p\right)-(0.48 D) q^{\nu}\left(\bar{n} \gamma_{\mu \nu} p\right)\right]\left(\overline{\nu_{L}} \gamma^{\mu} e_{L}\right)(42)
$$


The neutron beta decay rate depends linearly on the axial form factor at zero energy, i.e. $g_{A}$. In fact, $g_{A}$ is calibrated using this process. Since the Sagai-Sugimoto model overpredicts $g_{A}$ by about $2 \%$, it would also underpredict the neutron half-life by the same ratio.

In order to estimate the prediction of the Hard/Soft Wall model, we need to fix $D$. In principle, this could be done by using the physical value of either the axial form factor or the weak magnetism form factor. In the Sagai-Sugimoto model, these two form factors are successfully predicted as can be seen in Eq. (40). In the case of Hard Wall model, we find a tension between the two, that is if we choose to fit $D \simeq 0.94$ by $g_{A}^{e x p}=1.27$, then the magnetic form factor is too small by $50 \%$. There is however a chance for improving the bottom-up Hard Wall model, for which we hope to come back in the future [33].

(3) Parity non-conserving (PNC) pion-nucleon coupling

Our final example is something whose theoretical estimate has been difficult to obtain with other conventional tools. It is also a first non-trivial example which does not involve external leptons, so that the full aspect of our proposal should be used for its calculation. We are interested in the parity-violating couplings of mesons, especially the pions, to the nucleons induced by weak-interactions. For an illustrative purpose, we focus here only on the charged pion-nucleon coupling from a $W^{ \pm}$-exchange, and a more complete study of the problem will be presented elsewhere [33].

As before, the procedure of applying (8) and (201) to first order in $G_{F}$ starts by first solving, without $G_{F}$, the relevant normalizable modes for the particles involved in the process one is trying to study. Some of these normalizable modes, such as mesons, come from the bulk gauge fields of chiral symmetry, and the right-hand side of (8) or (20) is non-vanishing for them in general. These modes are then accompanied by the induced boundary values in the left-hand side of (8) or (20), which would act just like external sources for the chiral currents. From studying how external sources couple to the other particles of interest, we can obtain the $G_{F}$-induced interactions between the original modes and the other particles.

It is the pion in our case which carries an induced external source by (8) or (20), and how this external source would couple to the nucleons is described in the previous sections. In the Sakai-Sugimoto model, the pions are sitting in $A_{Z}$ as before and from (8), this entails a source

$$
A_{\mu}(+\infty)=-\frac{8 G_{F}}{\sqrt{2}} \sqrt{\frac{4 \kappa}{\pi}}\left(\partial_{\mu} \pi\right)=-\frac{8 G_{F}}{\sqrt{2}} f_{\pi}\left(\partial_{\mu} \pi\right)
$$


We have the same result in the Hard/Soft Wall model. Once we find the induced source, we can come straight to our previous results of nucleon couplings to external sources; (32) and (33) for the Sakai-Sugimoto model and similar expressions for the Hard Wall model. As we may need a general momentum transfer $q^{\mu}$ between the nucleons and the pions, we should solve the necessary $A^{T}\left(q^{2}\right)$ profile function numerically for a given $q^{2}$. For the $q^{2} \rightarrow 0$ limit however, we have a simplification as before to get

$$
\mathcal{L}_{N-\pi}^{\text {weak }}=-2 G_{F} f_{\pi}\left(\left(\bar{p} \gamma^{\mu} n\right)\left(\partial_{\mu} \pi^{+}\right)+g_{A}\left(\eta_{\mu \nu}-\frac{q_{\mu} q_{\nu}}{q^{2}}\right)\left(\bar{p} \gamma^{\mu} \gamma^{5} n\right)\left(\partial^{\nu} \pi^{+}\right)\right)+\text {h.c. }
$$

for the Sakai-Sugimoto model, and similarly for the Hard Wall model except the pionexchange term. Note that $\mathcal{F}_{\mu \nu}=0$ in our case as the source is purely longitudinal. Only the first piece in the above corresponds to the parity non-conserving (PNC) coupling, while the second term is a small correction to the usual $g_{A}$. The first term is identical to the contribution of pion exchange to the PNC coupling, as achieved by current algebra and chiral perturbation theory [34]. Although the above pion-nucleon coupling in $q^{2} \rightarrow 0$ limit is largely dictated by chiral algebra alone, its non-trivial $q^{2}$-dependence when we consider finite $q^{2}$ is beyond the ability of the chiral algebra. Our framework can give predictions for that. Our method can also provide predictions for the coupling of other excited mesons such as $\rho, \omega$, etc, whose details will be reported soon elsewhere [33].

\section{DISCUSSION}

We have outlined a proposal for including effective weak interactions in the framework of holographic QCD. By construction, this prescription should be applicable up to energies of a few $\mathrm{GeV}$. In order to demonstrate the implementation of the prescription, we chose two specific models, the Sagai-Sugimoto and Hard/Soft Wall models. The use of our method in three low- energy processes, namely charged pion decay, neutron beta decay, and PNC nuclear-meson couplings, showed the usefulness and strength of our prescription.

Contrary to other theoretical tools to calculate such reactions, e.g. chiral perturbation theory, the current approach not only recovers the operator structure of previous methods, but also gives a quantitative estimate, up to about 10-20\%, of the coupling constants. In addition, our weak-interacting holographic QCD can be extended to energies higher than the QCD scale, which is not assessable in other methods. 
Of course, there are many things to be done to improve the model. We hope that our first step will stimulate further study and possible refinement of our present proposal.

\section{Acknowledgments}

We thank Institute for Nuclear Theory (INT) at the University of Washington for the program "From Strings to Things: String Theory Methods in QCD and Hadron Physics", where this work was initiated, and H.U.Y. thanks the organizers for hospitality. D. G. is supported by DOE grant number DE-FG0200ER41132.

* E-mail: doron.gazit@mail.huji.ac.il

$\dagger$ E-mail: hyee@ictp.it

[1] T. Sakai and S. Sugimoto, Prog. Theor. Phys. 113, 843 (2005); Prog. Theor. Phys. 114, 1083 (2006).

[2] J. Erlich, E. Katz, D. T. Son and M. A. Stephanov, Phys. Rev. Lett. 95, 261602 (2005).

[3] L. Da Rold and A. Pomarol, Nucl. Phys. B 721, 79 (2005).

[4] A. Karch, E. Katz, D. T. Son and M. A. Stephanov, Phys. Rev. D 74, 015005 (2006).

[5] K. Ghoroku, N. Maru, M. Tachibana and M. Yahiro, Phys. Lett. B 633, 602 (2006).

[6] H. Boschi-Filho and N. R. F. Braga, Eur. Phys. J. C 32, 529 (2004); JHEP 0305, 009 (2003).

[7] T. Hambye, B. Hassanain, J. March-Russell and M. Schvellinger, Phys. Rev. D 74, 026003 (2006); Phys. Rev. D 76, 125017 (2007).

[8] E. Witten, arXiv:hep-th/0112258.

[9] H. U. Yee, Phys. Lett. B 598, 139 (2004).

[10] D. Marolf and S. F. Ross, JHEP 0611, 085 (2006).

[11] J. Erdmenger, N. Evans, I. Kirsch and E. Threlfall, Eur. Phys. J. A 35, 81 (2008).

[12] D. K. Hong, T. Inami and H. U. Yee, Phys. Lett. B 646, 165 (2007).

[13] D. K. Hong, H. C. Kim, S. Siwach and H. U. Yee, JHEP 0711, 036 (2007).

[14] M. Bianchi, D. Z. Freedman and K. Skenderis, Nucl. Phys. B 631, 159 (2002).

[15] D. K. Hong, M. Rho, H. U. Yee and P. Yi, Phys. Rev. D 76, 061901 (2007); JHEP 0709, 063 (2007). 
[16] J. Park and P. Yi, JHEP 0806, 011 (2008).

[17] K. Nawa, H. Suganuma and T. Kojo, Phys. Rev. D 75, 086003 (2007); Prog. Theor. Phys. Suppl. 168, 231 (2007).

[18] H. Hata, T. Sakai, S. Sugimoto and S. Yamato, arXiv:hep-th/0701280].

[19] G. F. de Teramond and S. J. Brodsky, Phys. Rev. Lett. 94, 201601 (2005).

[20] A. Pomarol and A. Wulzer, JHEP 0803, 051 (2008); arXiv:0807.0316 [hep-ph]].

[21] H. Hata, M. Murata and S. Yamato, arXiv:0803.0180 [hep-th]].

[22] K. Hashimoto, T. Sakai and S. Sugimoto, arXiv:0806.3122 [hep-th].

[23] K. Y. Kim and I. Zahed, arXiv:0807.0033 [hep-th].

[24] J. Polchinski and M. J. Strassler, Phys. Rev. Lett. 88, 031601 (2002).

[25] S. J. Brodsky and G. F. de Teramond, Phys. Lett. B 582, 211 (2004); Phys. Rev. D 77, 056007 (2008).

[26] S. Hong, S. Yoon and M. J. Strassler, JHEP 0604, 003 (2006).

[27] H. R. Grigoryan and A. V. Radyushkin, Phys. Rev. D 76, 115007 (2007).

[28] H. R. Grigoryan and A. V. Radyushkin, arXiv:0803.1143 [hep-ph].

[29] D. K. Hong, M. Rho, H. U. Yee and P. Yi, Phys. Rev. D 77, 014030 (2008).

[30] J. D. Walecka, "Theoretical Nuclear and Subnuclear Physics", Oxford Univ. Press, New York (1995).

[31] O. Bergman, S. Seki and J. Sonnenschein, JHEP 0712, 037 (2007); A. Dhar and P. Nag, JHEP 0801, 055 (2008); R. Casero, E. Kiritsis and A. Paredes, Nucl. Phys. B 787, 98 (2007).

[32] O. Aharony and D. Kutasov, arXiv:0803.3547 [hep-th]; K. Hashimoto, T. Hirayama, F. L. Lin and H. U. Yee, arXiv:0803.4192 [hep-th].

[33] D. Gazit and H. U. Yee, work in progress.

[34] See M. J. Musolf and S. A. Page, hep-ph/0601127 (2006) for a recent review.

[35] The "trace" here means the trace over $S U(N)$ color indices.

[36] Note that our convention is $A^{+}=\frac{1}{\sqrt{2}}\left(A^{1}-i A^{2}\right)$ with $A=A^{a} \frac{\sigma^{a}}{2}$ for bulk gauge fields, while $J^{+}=\left(J^{1}-i J^{2}\right)$ with $J^{a}=\bar{\psi} \frac{\sigma^{a}}{2} \psi$ for the boundary currents. Therefore, the source-operator coupling becomes $A^{a} J^{a}=\frac{1}{\sqrt{2}}\left(A^{+} J^{-}+A^{-} J^{+}\right)$in our convention.

[37] See however Ref. 28] for a 5D Chern-Simons term induced 3-point anomalous coupling.

[38] We performed the shift $N_{c} \rightarrow N_{c}+2$ for the coefficient of the last term that was argued in Ref.[15]. 
[39] See Ref.[31, 32] for attempts to introduce quark mass in the Sakai-Sugimoto model. 